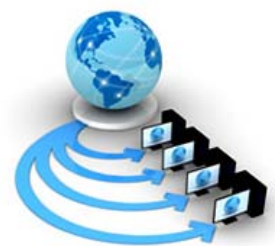

International Journal of Advanced Research in Computer Science

RESEARCH PAPER

\author{
Available Online at www.ijarcs.info
}

\title{
ANALYSIS OF UNICAST ROUTING IN COGNITIVE NETWORKS USING DDCR OVER TRADITIONAL NETWORKS
}

\author{
V.Lokeshwari Vinya \\ (Assistant professor, Dept. of CSE, GVPCE (A)), \\ Research Scholar, Dept. of I.T, GIT, GITAM University, \\ Visakhapatnam, India.
}

\author{
Dr. G.Venkateswara Rao \\ Associate Professor, Dept. of I.T, \\ GITAM Institute of Technology, GITAM University, \\ Visakhapatnam, India.
}

\author{
Rani Sesha Bhargavi \\ Assistant professor, Dept. of CSE \\ GVPCE (A), \\ Visakhapatnam, India.
}

\begin{abstract}
As there is rapid development of wireless and mobile communications, limited opening band is unable to meet the growing usage of mobile communication. In order to make better use of scarce spectrum resources, Cognitive Radio (CR) has been proposed to exploit underutilization portions of the spectrum, which is capable of interference sensing, environmental learning, and dynamic spectrum access. In this paper, a new routing algorithm for Cognitive Radio networks has been proposed. The algorithm is based on atomic time, known as DDCR (DillyDally Cognitive Routing). The DillyDally path is found by calculating the transmission delay of given packets on every link (weighted link). The transmission delays are found by using classic Dijkstra algorithm. Simulation results show the End-End delay of DDCR is less than that of the traditional routing algorithm. Thereby the network performance is improved.
\end{abstract}

Keywords: Spectrum, Transmission delay, Cognitive radio, Dijkstra’s, DDCR.

\section{INTRODUCTION}

Currently, wireless networks are assigned by government agencies according to a static assignment policy. In recent years, wireless applications has increased in request to regulatory authorities for spectrum allocation and its use in unlicensed bands, which cause overcrowding in the band. Recent studies by FCC highlight that many spectrum bands allocated through static assignment policies. To address this, Dynamic Spectrum Access (DSA) has been proposed [1] where the unlicensed spectrum users may use licensed band dynamically without any interference [2]. Devices with cognitive capabilities collected/combined to form a Cognitive Radio Networks (CRNS). Users in CRNs are classified into two types depending on utilization of spectrum bands: Primary users or licensed users (PUs) have priority in spectrum utilization and have license, Secondary users or unlicensed users (SUs) can interact without interfacing and dynamic manner. Research in this area mainly focus on lower layers OSI model that is physical layer and MAC layer [3].

In the CRNs network, each node acts as a Primary user (PUs), where the transmission is done within the specified spectrum. If PUs are not utilizing the spectrum, Secondary users (SUs) can occupy and utilize the spectrum by using spectrum sensing techniques. In addition, if PUs wants to utilize the spectrum, the SUs immediately exit from spectrum. In CRNs, interference situation is identified in terms of time frequency, availability of locations. The purpose of developing "Cognitive Routing Algorithm" is for finding the shortest path from source to destination in Cognitive radio network consisting of one or more channels.

In general, the main responsibility of network layer is routing, which is a process of selecting the best path in a network. Design issues of routing are store and forward packet switching, Services provided to the transport layer, implementation of connection-less services and implementation of connection-oriented services. In order to find an efficient path many techniques came into existence. These routing algorithms are classified into two types: Adaptive Routing algorithm, which is called as dynamic routing as updations are considered for each topology, Non-Adaptive algorithm that is considered static routing because its constant until it reaches the destination. Numerous techniques like link-state, distance vector, shortest path, Dijkstra's etc. has come into existence in order to find the efficient path. Consider fig1, which consist of different channels (nodes) with respective bandwidth's (weight assigned to a link). Here, $\boldsymbol{A}$ acts as source and $\boldsymbol{F}$ acts as destination. Shortest path $A-B-E-F$ is found using traditional algorithm whose cost is 5 , which is thereby considered to be the best path.

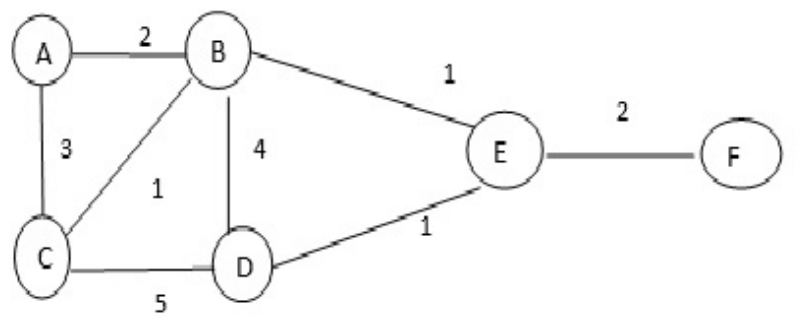

Fig 1: A simple network model

A weight for each links is defined to calculate the link capacity. Considering both weight and link capacity new algorithm is proposed to find smallest delay in a cognitive network. 


\section{SYSTEM OVERVIEW AND DESIGN}

Routing: The process of selecting a best path in a network is known as Routing, which is also a main design issue of network layer. Routing protocols such as IP, ICMP, IPsec, IPx, IGMP etc. are used to connect only computers but not mobile devices. Mobile Internet Protocol (MIP) has been proposed for communication amongst mobile devices. Later, communication among mobile devices took place through protocols such as HMIP, DHMIP, and HMMIP [4]. The selection of one of the protocol is decided based on the efficiency and performance, through which the user (source) is given access to the internet, which helps in transferring data (packets) to another user (destination). When the packets are transferred through a network, performance should be always under scrutiny. When most of the users are not utilizing the spectrum, it is considered as underutilized. In case, if all users or majorities of the users are using the spectrum, in such cases there may be scarcity of spectrum. To overcome this situation Cognitive networks has been proposed.

Cognitive networks: It is a technology used to solve the problem of spectrum deficiency by identifying vacant space in a spectrum and transmitting them, where vacant space is identified by spectrum sensing techniques and occupying vacant space is done by using dynamic spectrum access techniques. Generally users are classified are into licensed or authorized or primary users (PU's) and unlicensed or unauthorized or secondary users(SU's).In CRN's users does not have any spectrum band for their communication, therefore they are secondary users(Su's).In this case the common research issues are single-hop scenarios ,multi-hop scenarios, physical layer issues and MAC layer issues.

The major difference and challenges between routing in wireless networks and cognitive routing networks are unidirectional links, Link availability, Heterogeneous networks, Deafness problems etc. Routing protocols in CRN's are classified into Spectrum aware-based, Multi path-based, local coordination-based, Reactive source based and Treebased [5].

Spectrum Sensing: The main challenge in CRN's network is secondary user (SU) is need to detect whether primary user (PU) is using the spectrum or not. If PU is not using ,SU occupies and similarly when SU is using,PU want that spectrum SU needs the spectrum SU has to exit.to address this situation a technique called Spectrum sensing has been introduced. Spectrum sensing and estimation is the first step to implement first Cognitive Radio system techniques such as Non-Cooperative system, Cooperative system and Interference based schemes can be used [5].

Single-hop and Multi-hop: Hop refers to link between one device to another. Hop count refers to numbers of intermediate devices between source to destination data has to be transmitted.in case, there is only one hop between source to destination in a network then it is known as Single-hop. If there are multiple hops between source to destination in an network is referred as multi-hop.

Weighted link(s): In a network, the nodes are connected either by direct links or by indirect link.to each and every link a cost is assigned. The link that has minimum cost is called best link or weighted link.

Problem Statement: The spectrum allocation request has increased proportionally in the recent years due to the success of wireless applications.
- There has been abundant range of applications developed in various fields, which has caused excessive usage of unlicensed band.

- Also, the spectrum bands which are licensed are not even and always depend on few wireless technologies who have good market expansion and their trading success to which there are assigned frequencies.

Drawbacks:

- Most of the spectrum has not fully utilized

- The average utilization of such bands varies between $15 \%$ and $85 \%$

- More latency on packet delivery and processing load for routers.

- Delay before a sending and after problems arose.

- $\quad$ Packet header size grows with length of the route.

\section{Proposed system:}

The problem statement can be addressed with the help of DSA-Dynamic Spectrum Acess.With the help of DSA,the spectrum bands which are licensed can be used by unlicensed users either dynamically or without interference.

- Cognitive Radio (CR) technology is an agile solution for spectrum congestion and spectrum access utilization problems.

- $\quad$ CR technology can exploit unused licensed band to meet the increasing demand for radio frequency.

- Communications in CRN'S are operative to access and perform operations.

- To provide dynamic access to spectrum. Advantages in Proposed System:

- Improve Quality of Service (QOS) [6]

- $\quad$ Overcome Radio spectrum scarcity

- Avoid intentional radio jamming scenarios

- $\quad$ Benefits to the service provider

- Provides an efficient means of measuring and reporting usage.

In the proposed algorithm active probability, packet length and number of channels are considered inputs, which are used to calculate the transmission delay in-between the communication links for a specific channel. The resultant transmission delay is assigned as modified weights to the respective communication links.

FUNCTIONAL REQUIREMENTS: Functional Requirements is also known as Behavioral requirements of a system because it defines what inputs are to be provided, what outputs are to be expected, how to process the data to get the solution. The following are the functional requirements for the proposed system:

- How to construct full network model with multiple nodes.

- Describing how to calculate packet length.

- Assigning weights to every communication link.

- Checking probability.

- Number of channels.

NON-FUNCTIONAL REQUIREMENTS: For any system these has to be contain qualities that specify the criteria, which can be used to justify the operation of a system, rather than the behavior. These qualities are generally referred as nonfunctional requirements.

Route to Destination: Generally, the routing architecture varies when the packet transfers from lone source to lone destination and lone source to multiple destinations. When the 
packets are transferred from lone source to lone destination, the routing architecture is simple. Whereas, in case of single source to multiple destinations, the routing architecture is little complex.

Routing is Assured: Any routing services has to be assured to both sender and receiver. The assurance is given to the parties through messages. If packet has been successfully delivered, both sender and receiver have to be notified that a specific packet has been sent to and received from to the respective parties. In contrary, if there is delay in packet transmission or packet loss, the sender and receiver has to be given necessary information about the tracking details of a specific packet.

\section{ABOUT NS TOOL:}

NS (Network Simulator) is a Software tool used for designing and to analyze the performance of a real-time network, where the networks are implemented with devices, links, routers etc.

At present in many technologies like mobile ad hoc networks, wireless LAN's, Cognitive radio networks, wireless sensor networks etc. are designed and analyzed with simulators. NS-2, NS-3, Netsim, OMNET++, REAL, QualNet, J-Sim etc. are latest simulator tools.

Proposed system is implemented with NS2 simulator, where NS2 is developed with C++, TCL and OTCL.Here, for programming in NS2 TCL scripting is used. Additionally C++ is also used for adding modules if required.

1. Programming languages such as $\mathrm{C}++$ and Python is used for building the scripting capability of NS-2. pybindgen library in python is used to wrap the NS2 library, which parse the NS2 C++ headers to gccxml and pygccxml, which in burn generate $\mathrm{C}++$ files compiled to the NS2 python module. Special feature such as attribute based systems manage (default and) the simulation parameters which are considered to be pre-instance values.

2. Work flow of network simulator: The following steps are used for creating networks that have to be simulated:

i) The foremost step is to define topology, i.e. to create the basic facilities and interconnections in between networks. To facilitate this process NS2 has a system of containers and helpers.

ii) Modes such as TCP, UDP, IPV4, one-to-one devices are added to the simulation so that a network model is developed.

iii) Attribute system is used to set the node and link configurations, where the adjust values are set.

For example:

To create a node: set $\mathrm{n} 0$ [\$ns node]

To provide link between nodes: \$ns duplex-link \$n0 \$n1 2Mb 10ms Drop Tail

iv) Packet transmission can be visualized through generating the events with help of data provided by the user.

v) The result of the simulation is a horodatage trace to give any further information the data can be analyzed with tools such as R.

vi) Pictorial representation such as graphs can be drawn using the resultant data with the help of tools such as XGraph, matpotlib, Gnuplot, MS-Excel etc.

\section{IMPLEMENTATION OF THE SYSTEM}

\section{Proposed Algorithm:}

Step1: Create a network by using set of nodes i.e. $G=(V, E)$ whereas $\mathrm{V}$ nodes/vertices i.e. $\mathrm{V}=\{\mathrm{V} 1, \mathrm{~V} 2, \ldots, \mathrm{Vn}\}, \mathrm{E}$ is the links/edges i.e. $\mathrm{E}=\{\mathrm{E} 1, \mathrm{E} 2, \ldots . \mathrm{En}\}$.

Step2: Initialize weight (w) for each link as $\{1,2,3 \ldots\}$

For example: V1->V2=1, V1->V3=4 etc.

Step3: Let us assume initial channel probability as XPV1 $=0.82$. So to calculate the other links XPV2, XPV3,..., XPVN.

Step4: Assume Packet length as ' $\mathrm{L}$ '.

Step5: calculate link capacity by using Shannon's theorem followed by calculating channel capacity as:

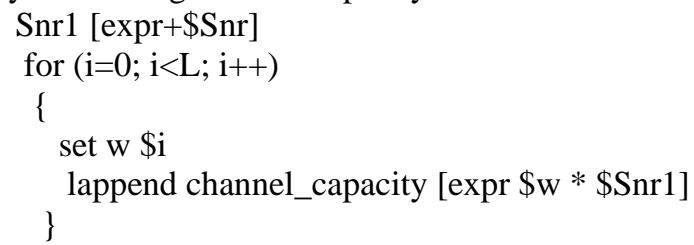

Step6: calculate the link capacity as:

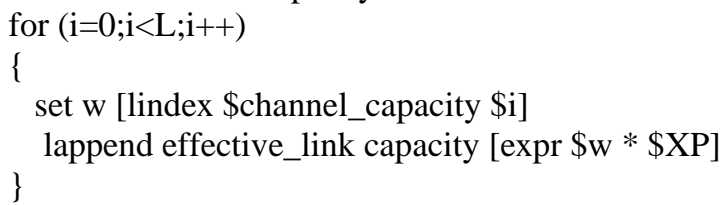

Step7: Calculate Transmission delay as:

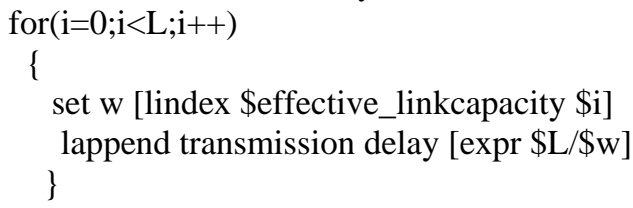

Step8: Finally assign the resultant transmission delay as input to traditional technique (i.e. Dijkstra's) to find out the DD path.

\section{EXPERIMENTAL RESULTS}

\section{Screenshots:}

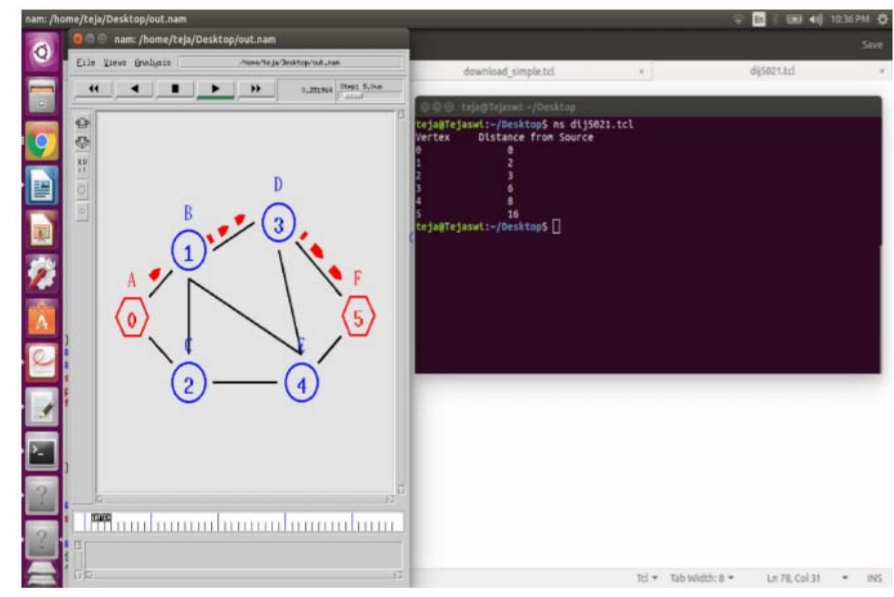

Figure 4.1: Transmission delay using Traditional Algorithm 


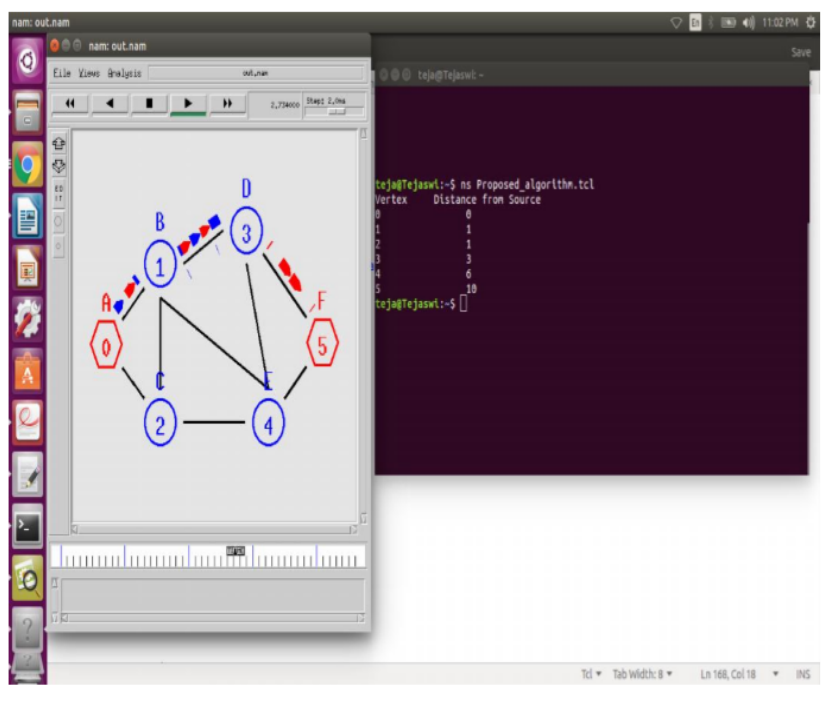

Figure 4.2: Transmission delay using Dillydally algorithm.

\section{Graph Analysis:}

\begin{tabular}{c|c|c}
\cline { 2 - 3 } \multicolumn{2}{c|}{} & \multicolumn{2}{c}{ Source is '0' } \\
\hline Desination & $\begin{array}{r}\text { Traditional Algorithm } \\
\text { (Dijkstra Algorithm) }\end{array}$ & Proposed Algorithm \\
\hline 0 & 0 & 0 \\
\hline 1 & 2 & 1 \\
\hline 2 & 3 & 1 \\
\hline 3 & 6 & 3 \\
\hline 4 & 8 & 6 \\
\hline 5 & 16 & 10 \\
\hline
\end{tabular}

Figure 4.3: Traditional Vs. Proposed

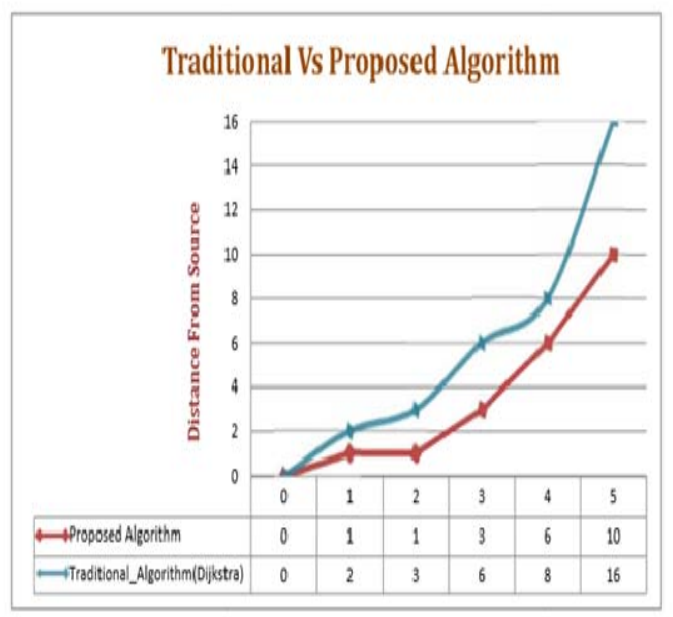

Figure 4.4: Analysis between Traditional and Proposed techniques.

\section{CONCLUSION}

To reduce the problem such as deficiency of spectrum and to increase the un-utilized space in a network, Cognitive radio technique has been came into existence. Many routing techniques such as Dijkstra's have been reviewed to implement cognitive networks. But in vain there is no utilization of spectrum. To overcome this problem, the proposed DDCR algorithm is applied on traditional algorithms using NS2 simulator on a single networks. It is observed that efficiency is comparatively improved using DDCR algorithm than the traditional ones.

\section{REFERENCES}

[1] Moustafa Youssef, Mohamed Ibrahim, Mohamed Abdelatif, Lin Chen and Athanasios V.Vasilakos,"Routing Metrics for Cognitive Radio Networks: a survey”, IEEE, Vol 16, First quarter 2014.

[2] Matteo Cesana, Francesca Cuomo,Eylem Ekici, "Routing in cognitive radio networks: Challenges and Solutions”, Elsevier, adhoc networks,2010.

[3] Claudia Cormio, Kaushk R.Chowdhury, "A survey on MAC protocols for cognitive radio networks”, Elseiver, ad-hoc networks, 2009.

[4] Nadjia Kara, "Mobility management approaches for mobile IP networks: performance comparisons and use recommendations”, IEEE, Vol. 8, No. 10, October 2009.

[5] Mansi Subhedar, Gajanam Birajdar, "Spectrum sensing techniques in Cognitive radio networks", IJNGN, Vol.3, No.2, June 2011.

[6] http://ieeexplore.ieee.org/document/6845054/ 